\title{
Excision of A Rare Bilateral Malignant Carotid Paraganglioma: What We Have Done and What's to Do
}

\author{
Grasso Marica*1, Costigliola Luciana ${ }^{2}$, Ciorra Francesca Romana ${ }^{2}$, De Sena Gabriele ${ }^{3}$, Esposito MG $^{4}$ \\ and Puzziello Alessandro ${ }^{1}$ \\ ${ }^{1}$ Faculty of Medicine, Surgery and Dentistry, University of Salerno. Salerno, Italy \\ ${ }^{2}$ UOC General Surgery, "A Cardarelli" Hospital. Naples, Italy
}

${ }^{3}$ Faculty of Medicine and Surgery, "Luigi Vanvitelli" University of Campania. Naples, Italy

${ }^{4}$ UOS Endocrino surgery, Ospedale del Mare. Naples, Italy

*Corresponding author: Marica Grasso, Professor (Assistant) Doctorate student, Faculty of Medicine, Surgery and Dentistry, Via S.

Allende, 84080 Baronissi, Salerno, Italy

\section{ARTICLE INFO}

Received: 慧 January 18, 2019

Published: 慧 February 04, 2019

Citation: Grasso M, Costigliola L, Ciorra FR, De Sena G, Esposito MG, Puzziello A. Excision of A Rare Bilateral Malignant Carotid Paraganglioma: What We Have Done and What's to Do. Biomed J Sci \& Tech Res 14(1)-2019. BJSTR. MS.ID.002483.

\section{ABSTRACT}

Paragangliomas are non-epithelial neuroendocrine neoplasms. They are most commonly identified in the head and neck, most frequent in the carotid body, but only about 5\% of carotid paragangliomas are bilateral. Surgery is an effective treatment although it may be difficult in patients with the advanced types. Here we present a rare case of bilateral malignant carotid paraganglioma in a female patient treated with two-step surgery and life-long surveillance.

Keywords: Paraganglioma; Carotid neoplasm; Non-epithelial neuroendocrine neoplasm; Head and neck, surgery

\section{Introduction}

Paragangliomas (PGL) are non-epithelial neuroendocrine neoplasms (NEN) [1] that derive from paraganglia, which are seen in close association with components of the sympathetic and parasympathetic nervous systems [2,3]. They can arise in any location where paraganglia normally reside [4]. Because of widespread distribution of paraganglia, paragangliomas can occur at virtually all locations in the body except within the brain and in bone although they have been reported in the extremities [5]. They are most commonly identified in the head and neck, being most frequent in the carotid body, followed by jugulo tympanic paraganglia, vagal nerve and ganglium nodosum, as well as laryngeal paraganglia. Abdominal sites include the well-known urinary bladder neoplasms that originate in the Zuckerkandl organ. However, other unusual sites of origin include peri-adrenal, paraaortic, inter-aortocaval, and paracaval retroperitoneal sites, as well as neoplasms in organs where they may not be expected such as thyroid, parathyroid, pituitary, gut, pancreas, liver, mesentery, lung, heart and mediastinum. Carotid body neoplasms represent approximately $65 \%$ of all head and neck paragangliomas and only about $5 \%$ of carotid body neoplasm are bilateral. In the literature, most of the cases presented concern benign and unilateral neoplasms. We present here a rare case of bilateral malignant carotid paraganglioma in a female patient.

\section{Case Report}

A 40-years-old woman was admitted to the $2^{\text {nd }}$ division of General Surgery Department of "A. Cardarelli" Hospital of Naples with a 15-years history of bilateral neck swelling and diagnosis of bilateral lymphadenopathy. She denied any history of similar swelling elsewhere in body. There was no history of similar swelling in any family members. There was no history of fever, weight loss or loss 
of appetite and no other constitutional symptoms were described such as episodes of palpitations or hypertension. Physical examination was negative except for bilateral neck masses with pulsation. The masses were firm with free mobility. Results of cranial nerve examinations were normal and the tests for pheochromocytoma were negative. Several ultrasound examinations had been repeated over the years always highlighting the presence of two irregular solid nodules of soft tissue density, measuring $3.7 \mathrm{~cm}$ and $1.7 \mathrm{~cm}$ located in the right and left carotid artery bifurcation, respectively. Additionally, RM examination, performed 2 years before the hospitalization, revealed heterogeneous reinforcement in the bilateral carotid sheath area, however the boundary between the masses and right and left carotid arteries was clear.

Due to a significant increase in the size of the mass located in the right carotid, revealed by ultrasound examination (Figure 1), the patient referred to our hospital. The patient underwent surgery for the removal of right-side lesion and was discharged the day after. Although the encapsulated mass was strongly vascularized and adherent to multiple enlarged lymph nodes, it was dissected from the carotid bifurcation, and the common carotid, internal carotid and external carotid arteries remained intact. The resected specimen was a solid, pink, and well circumscribed mass. Histological analysis revealed the rich vasculature and numerous reactive lymph node were found. According to immunohistochemical staining, negative for keratin, positive for chromogranin, synaptophysin and CD56 and sustentacular cells stained positively for S100 protein a diagnosis of right carotid paraganglioma was made. Proliferation index reported was less of 4\%. According to immunohistochemical diagnosis patient was submitted to a novel neck ultrasound and angio-RM examinations in order to re-evaluate the left side mass.

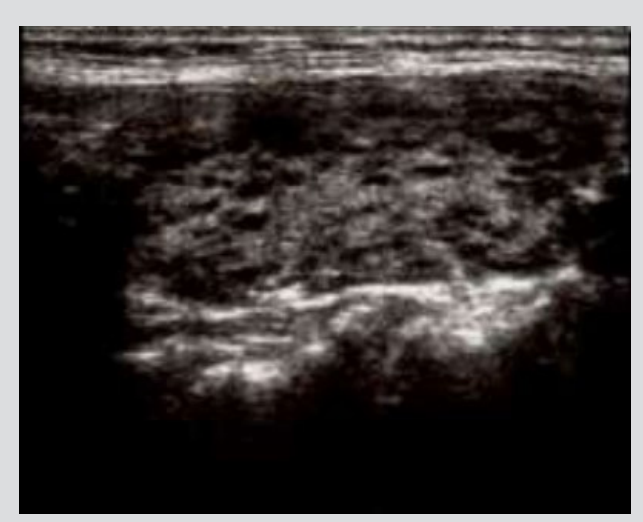

Figure 1.

The subsequent examinations revealed the left-side lesion had the same characteristics of hypervascularization of the removed one and the presence of numerous reactive lymph nodes, while remaining the same size as the previous controls. The patient underwent surgery for the removal of left-side paraganglioma and was discharged the day after. In this case the mass was strongly vascularized and adherent to multiple enlarged lymph nodes too.
In the same way immunohistochemical staining was negative for keratin, positive for chromogranin, synaptophysin and CD56 and sustentacular cells stained positively for S100 protein with proliferation index around 5\%. Two months after the second operation, the patient underwent TC total body scan for follow-up. The result was negative for lymph node and distant metastasis, while on the right carotid the appearance of a new lesion of $7 \times 8 \times 12 \mathrm{~mm}$ has been highlighted. The patient refused surgery for the right-side recurrence considering the post-operative complication risks. At the time of writing this report, the patient is still being followed-up.

\section{Discussion}

The diagnosis of paraganglioma (PGL) can be simple when the lesion is in an expected location and when the patient presents with classical symptoms related to catecholamine excess. However, the diagnosis is often missed when they occur in unusual locations or when there is lack of symptomatology. Imaging often reports the presence of lymphadenopathy in those patients and this can be misdiagnosed on imaging as lymphoma or metastatic carcinoma in lymph nodes. In addition, according to some authors paragangliomas should no longer classified as benign [6], since even without metastatic spread, multifocality or progressive disease they can have significant morbidity and mortality, especially in familial forms, due to the presence of a specific gene mutation $[7,8]$. More important, so is to document the size, location and mutation status of a lesion in fact awareness of the existence of PGLs in unusual locations and the likelihood of multifocal primary neoplasms in patients with germline genetic predisposition to this disease will reduce errors in diagnosis and provide more accurate data collection to allow progress in the understanding of these neoplasms $[9,10]$. An ultrasound study is a rapid, convenient and non-invasive exam that may be used to detect the margin, vascularity and invasion of a mass, and any regional lymph node metastasis [11].

Ultrasound is more useful for screening familial cases and follow-up procedures while a Doppler analysis of the mass is useful to evaluate intratumor blood flow and is valuable in differentiating paragangliomas from other solid, non-hyper vascularized masses [12]. Contrast ultrasonography may also aid the evaluation of the blood supply to the neoplasm [13]. Therefore, the ultrasound is a suitable technique for the identification of a Carotid paraganglioma and the possible diagnosis of carotid paraganglioma may be anticipated when a solid mass is detected at the carotid bifurcation, however, is unable to determine whether the mass is benign or malignant. Ultrasound is also limited due to an inability to identify deeply located lesions [12]. Immunohistochemistry plays a key role in confirming the diagnosis. Many pathologists believe that the stains required are chromogranin, synaptophysin and S100; the first two yield cytoplasmic positivity in neoplasm cells whereas the demonstration of S-100-positive sustentacular cells encircling neoplasm nests is highly characteristic of paragangliomas. The 
fallacy is that any type of NEN can stain for synaptophysin and chromogranin, and S100-positive sustentacular cells occur in neuroendocrine tumors (NET) at many locations.

It is therefore important to identify other biomarkers that allow the distinction from NET. In this regard, the importance of keratins cannot be overemphasized and the identification of a NEN that lacks keratin should prompt consideration of this diagnosis [14]. However, there are no accepted immunohistochemical markers for the distinction between malignant and benign paraganglial neoplasms. It is usually determined to be malignant only in case of recurrence or if metastasis is established in the lymph nodes or distant organs $[15,16]$. The traditional treatment of paraganglioma is surgery to the primary site and neck dissection to address regional disease. Surgery followed by adjuvant radiation is used less frequently, and chemotherapy is typically reserved for distant disease [17]. Even if surgical resection is the recommended treatment for paraganglioma, with the main goal of excising the neoplasm and preventing local advancement, for patients with bilateral mass, surgery may not be performed all at once to avoid serious complications including cranial nerve dysfunction: approximately $10 \%$ of the cases had nerve injury of the hypoglossal, glossopharyngeal, recurrent-laryngeal, or spinal accessory nerves, or involvement of the sympathetic chain [18].

\section{Conclusion}

Surgery is an effective treatment for patients with carotid paragangliomas. Although, it may be difficult in patients with the advanced types, in experienced hands, complication rates are very low [19]. Malignant forms can also be treated successfully if surgery is followed by neck dissection for lymph node metastases or chemotherapy for distant metastases. Although the 5-year overall survival after surgery reported in the literature for malignant paraganglioma ranges from $40 \%$ to $95.4 \%$ [20], the individual prognosis is unpredictable and thus life-long surveillance is mandatory

\section{References}

1. Rindi G, Klimstra DS, Abedi-Ardekani B, Asa SL, Bosman FT, et al. (2018) A common classification framework for neuroendocrine neoplasms: An international Agency for Research on Cancer (IARC) and World Health Organization (WHO) expert consensus proposal. Mod Pathol 31(12): 1770-1786.

2. Oudijk L, de Krijger RR, Pacak K, Tischler AS (2016) Adrenal medulla and extra-adrenal paraganglia. In Endocrine Pathology; Mete O, Asa SL (Eds.), Cambridge University Press: Cambridge, UK, pp. 628-676.

3. Hayashi T, Mete $O$ (2014) Head and neck paragangliomas: What does the pathologist need to know? Diagn Histopathol 20(8): 316-325.

4. Tischler AS, Asa SL (2018) Paraganglia. In: Histology for Pathologists; Mills SE Ed; Wolters Kluwer: Philadelphia, PA, USA.
5. Chow LTC, Chan MHM, Wong SKC (2018) Functional Ulnar Nerve Paraganglioma: First Documented Occurrence in the Extremity with Hitherto Undescribed Associated Extensive Glomus Cell Hyperplasia and Tumorlet Formation. Int J Surg Pathol 26(1): 64-72.

6. Kimura N, Capella C, De Lellis RA (2017) Extra-adrenal paragangliomas. In WHO Classification of Tumours of Endocrine Organs, ( $4^{\text {th }}$ edn), Lloyd RV, Osamura RY, Kloeppel G, Rosai J (Eds.), International Agency for Researchon Cancer: Lyon, France 10: 190-195.

7. Young AL, Baysal BE, Deb A, Young WF Jr (2002) Familial malignant catecholamine-secreting paraganglioma with prolonged survival associated with mutation in the succinate dehydrogenase B gene. J Clin Endocrinol Metab 87(9): 4101-4105.

8. Hammer S, Jansen JC, van der Kleij-Corssmit EP, Hes FJ, Kruit MC (2012) Case of spontaneous regression of carotid body tumor in a SDHD mutant: A discussion on potential mechanisms based on a review of the literature. World J Surg Oncol 10: 218.

9. Assadipour Y, Sadowski SM, Alimchandani M, Quezado M, Steinberg SM, et al. (2017) SDHB mutation status and tumor size but not tumor grade are important predictors of clinical outcome in pheochromocytoma and abdominal paraganglioma. Surgery 161: 230-239.

10. Ayala-Ramirez M, Feng L, Johnson MM, Ejaz S, Habra MA, et al. (2011) Clinical risk factors for malignancy and overall survival in patients with pheochromocytomas and sympathetic paragangliomas: Primary tumor size and primary tumor location as prognostic indicators. J Clin Endocrinol Metab 96: 717-725.

11. Grasso M, Puzziello A, De Palma M (2019) Preoperative ultrasound evaluation of laterocervical lymph nodes: timing and experience modify the treatment of patients with differentiated thyroid cancer.

12. Pacheco-Ojeda L (2001) Malignant carotid body tumors: report of three cases. The Annals of otology, rhinology, and laryngology. 110: 36-40.

13. Arslan H, Unal O, Kutluhan A, Sakarya ME (2000) Power Doppler scanning in the diagnosis of carotid body tumors. J Ultrasound Med 19: 367-370.

14. Singh S, Asa SL, Dey C, Kennecke H, Laidley D, et al. (2016) Diagnosis and management of gastrointestinal neuroendocrine tumors: An evidencebased Canadian consensus. Cancer Treat. Rev 47: 32-45.

15. Torrealba JI, Valdes F, Kramer AH, Mertens R, Bergoeing M, et al. (2016) Management of Carotid Bifurcation Tumors: 30-Year Experience. Annals of vascular surgery 34: 200-205.

16. Moskovic DJ, Smolarz JR, Stanley D, Jimenez C, Williams MD, et al. (2010) Malignant head and neck paragangliomas: is there an optimal treatment strategy? Head Neck Oncol 2: 23.

17. Hinerman RW, Amdur RJ, Morris CG, Kirwan J, Mendenhall WM (2008) Definitive radiotherapy in the management of paragangliomas arising in the head and neck: a 35-year experience. Head Neck 30: 1431-1438.

18. Bastounis E, Maltezos C, Pikoulis E, Leppaniemi AK, Klonaris C, et al. (1999) Surgical treatment of carotid body tumours. The European journal of surgery = Acta chirurgica 165: 198-202.

19. Conzo G, Avenia N, Ansaldo G, Calò P, De Palma M, et al. (2017) Surgical treatment of thyroid follicular neoplasms: results of a retrospective analysis of a large clinical series. Endocrine 55(2): 530-538.

20. Sethi RV, Sethi RK, Herr MW, Deschler DG (2013) Malignant head and neck paragangliomas: treatment efficacy and prognostic indicators. Am J of otolaryng 34: 431-438. 


\section{ISSN: 2574-1241}

DOI: 10.26717.BJSTR.2019.14.002483

Marica Grasso. Biomed J Sci \& Tech Res

(C) This work is licensed under Creative

Submission Link: https://biomedres.us/submit-manuscript.php

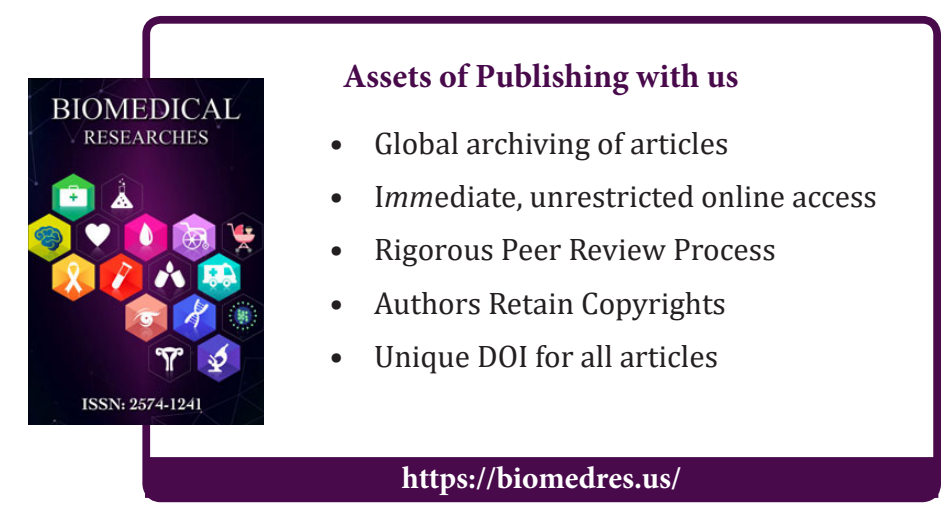

